Dominika Dykta

Università della Slesia, Katowice

Polonia

(D) https://orcid.org/0000-0003-3685-1782

\section{Come si esprimono le emozioni durante il cambio di codice dall'italiano al dialetto nella comunità talamonese}

How emotions are expressed during the change of code from Italian to dialect in the Talamonese community

\begin{abstract}
The aim of the article is to show how Italians express their emotions when changing the code from Italian to dialect on the example of the Talamona's dialect. At the beginning, it was presented what emotions are and in which categories they should be considered, the specificity of the Talamona's dialect and what the change of code is. The theory is supported by examples of change of code from Italian to dialect. The result of the work is to show that the respondents from Talamona very often change the code from Italian to dialect due to their emotionality. The article introduces the concept of change of code and shows how emotionality affects it.
\end{abstract}

\title{
Keywords
}

Dialect, emotions, change of code, Italian language, sociolinguistic

In questo studio si andranno a esaminare le emozioni che vengono dimostrate in quanto uno dei fattori che provoca il cambio di codice tra la lingua italiana e la varietà dialettale. Durante le analisi del dialetto talamonese, ossia l'analisi cognitiva dell'alternanza e del cambio di codice tra il dialetto e la lingua italiana, è stato notato che un dialettofono cambia la lingua dall'italiano al dialetto o viceversa nelle varie situazioni, ad esempio quando il dialetto svolge una funzione precisa, quando il dialettofono parla con qualcuno la cui competenza comunicativa è maggiore in dialetto, a causa dell'abitudine linguistica, e infine, quando esprime le emozioni. Questi risultati sono stati pubblicati recentemente (Dykta, 2021). In 
questo articolo, invece, un'attenzione particolare verterà sugli aspetti relativi alle emozioni, le quali provocano l'uso del cambio di codice.

\section{Emozioni}

La discussione dovrebbe partire dal chiarimento di come si dovrebbero comprendere le emozioni. Come punto di riferimento è stata presa la teoria dell'approccio multicomponente alle emozioni. Dąbrowski (2014) ha constatato che nella soluzione multicomponente, le emozioni sono gli stati psicofisici del sistema mente- cervello- corpo. In quanto tali, da un lato, hanno un carattere mentale, e dall'altro, corporeo. In altre parole, consentono il contatto con la realtà esterna e aiutano a percepire la realtà, essendo un certo tipo di risposta dell'organismo umano alle situazioni d'ogni giorno. Va tenuto presente che le emozioni sono fenomeni complessi e quindi non possono essere ridotte soltanto all'aspetto fisiologico, cognitivo o comportamentale, ma piuttosto dovrebbero essere definite in quanto stati cognitivi, correlati con i cambiamenti fisiologici e corporei, quindi anche neurologici, possedenti una sfumatura emotiva e che stimolano gli uomini ad agire (A. Dąbrowski, 2014: 130).

Avendo spiegato come si comprendono le emozioni, si passa ai possibili modi di suscitarle. Ekman (2012) distingue appunto nove modi di suscitare le emozioni, tra le quali abbiamo la valutazione automatica, la valutazione volontaria, i ricordi, soprattutto delle esperienze emotive, l'immaginazione, la conversazione su eventi emotivi passati, l'empatia, l'atteggiamento e le istruzioni di altre persone, l'infrangere delle regole sociali, la particolare espressione del viso.

Ora è opportuno parlare dell'aspetto cognitivo delle emozioni. È importante enfatizzare che le emozioni hanno decisamente un carattere cognitivo, il quale è responsabile del riconoscimento delle informazioni e della loro valutazione. Prima nella mente umana si identificano gli oggetti, gli eventi e poi vengono valutati. Si presuppone allora l'esistenza dei concetti e delle credenze, la cui fonte sono i processi cognitivi, come la percezione o la memoria. La valutazione può essere automatica, immediata, inconscia, quindi fisiologica, la quale riguarda ciò che troviamo nell'ambiente buono o sfavorevole. Bisogna allora sottolineare che la valutazione è a volte solo automatica e inconsapevole, mentre talvolta è pienamente cosciente e in un certo senso precede l'emozione. Partendo da questo presupposto, ci si rende conto che le emozioni tendono ad essere degli stati coscienti, ma possono anche verificarsi senza consapevolezza. Grazie alle emozioni, una persona sa se farsi coinvolgere in qualche faccenda o ritirarsi. Da ciò si può evincere che quando si ha paura, di solito la prima reazione consiste nello scappare, perché quando si ha a che fare con un fenomeno nuovo, sconosciuto, esso può essere 
inquietante e semplicemente può spaventare. A questo proposito occorre rimarcare il fatto che esiste una divisione delle emozioni in quelle positive, piacevoli ad esempio la soddisfazione, l'innamoramento, la gioia, la simpatia e quelle negative, spiacevoli come la tristezza, la delusione, l'irritazione, il disgusto. Di solito, l'uomo si sforza per ottenere il piacere ed evita il dolore. Succede che le emozioni negative non devono essere vissute come spiacevoli, ad esempio per alcuni, la vendetta può essere piacevole (A. Dąbrowski, 2014).

\section{Cambio di codice}

Conviene ora volgere l'attenzione al cambio di codice, un fenomeno sociolinguistico verificatosi quando un parlante bilingue cambia la lingua del discorso dall'italiano in un'altra varietà. Un'osservazione pertinente è che il cambio di codice appare, mentre la conversazione inizia con una lingua, poi cambia il destinatario, ad esempio entra una terza persona, oppure viene cambiato l'argomento, e in seguito, il parlante modifica la varietà che usa e passa dall'italiano al dialetto o viceversa. È più spesso osservato il cambio di codice che l'alternanza (C. Grassi, A. A. Sobrero, T. Telmon, 2010).

Avendo introdotto anche il termine di alternanza bisogna spiegare in che cosa consiste la divisione degli usi alternati, sistematizzata da Grassi, Sobrero, Telmon (2010, 2012). Gli usi alternati si dividono in alternanza di codice e in cambio di codice. Invece il cambio di codice si divide in commutazione di codice, enunciato mistilingue, prestito e code - shifting. In questo articolo, l'attenzione viene posta soltanto sul cambio di codice, precisamente sulla commutazione di codice.

Un'osservazione cruciale è che i fenomeni descritti non sono soltanto presenti in Italia, ma in ogni paese dove ci sono più lingue. Bettoni e Rubino (1996) analizzarono i casi degli emigrati siciliani e veneti che sono in Australia, specialmente i loro cambi tra i codici italiano - dialetto - inglese secondo i domini, come scuola, lavoro, negozi, amici ecc. Bisogna mettere in risalto il fatto che Grassi, Sobrero, Telmon $(2010,2012)$ scrivevano a favore dell'accettazione comune di questi fenomeni sociolinguistici che potrebbero aiutare a sopravvivere il dialetto in Italia, perché secondo l'ISTAT, l'uso del dialetto in Italia diminuisce.

Come si è già accennato, la commutazione di codice, chiamata anche il codeswitching è

il passaggio (dal punto di vista del processo, naturalmente; volendo accentuare il punto di vista del risultato, si dirà 'giustapposizione') nel discorso da un sistema linguistico a un altro sistema linguistico in concomitanza con un cambiamento nel flusso della situazione comunicativa; un passaggio funzio- 
nale da un codice o da un sistema linguistico all'altro, all'interno di uno stesso evento o episodio comunicativo (G. Berruto, 1990: 108).

Bisogna tener presente che questo fenomeno, di solito, avviene alla fine di una frase e perciò è chiamato interfrasale.

La commutazione di codice dipende dalla provenienza dell'utente di lingua se egli viene e abita in città o in campagna, dagli aspetti generazionali o socioculturali. Meriterà inoltre un cenno il fatto che la commutazione di codice è intenzionale, dato che è legata alla concreta intenzione del parlante di comunicare qualcosa, ossia il proprio coinvolgimento, il rapporto informale e la consuetudine della situazione comunicativa (G. Gobber, M. Morani, 2010).

Per di più, molti linguisti si interessavano di questo fenomeno (Alfonzetti, 1992; Berruto, 1985, 1989, 1990; Grassi, 2001; Grassi, Sobrero, Telmon, 2010, 2012; Sobrero, Miglietta, 2009) e sono giunti a diverse conclusioni. Berruto (1985) lo analizzava a Torino e sosteneva che esso avveniva per incomprensione dell'enunciato da parte dell'interlocutore, l'uso del dialetto allo scopo di sentirsi a suo agio. Elencava anche le funzioni del fenomeno, quali routine (saluti, espressioni di cortesia, ecc.), enfasi emotiva (interiezione, abitudine, battuta, ripetizioni, conferma), funzione discorsiva, sviluppo narrativo o argomentativo del discorso, conclusione. Secondo lo studioso, "l'italiano tende a esser usato di più in funzione di "riporto al pubblico" e il dialetto in funzione di "riporto al privato"' (G. Berruto, 1985: 66-75). La considerazione cruciale da fare è quella che Berruto ha notato l'aspetto emotivo del cambio di codice, quando il locutore esprime interiezioni, battute, ripetizioni, conferme o abitudini.

Passando ora allo studio di Gumperz (1982), bisogna dire che egli distingueva il code-switching situazionale e il code-switching metaforico e faceva opposizione fra we-code e they-code. Secondo il ricercatore, la commutazione di codice porta un significato sia linguistico che sociale. Lo studioso ha analizzato la lingua hindi e i dialetti in India e ha notato che i locutori eseguivano la commutazione

per rivolgersi agli alfabetizzati e anche agli analfabeti, per trasmettere un significato preciso, per facilitare la comunicazione, per negoziare con maggiore autorità, per catturare l'attenzione stilistica, emotiva, enfatica, per sottolineare un fatto, per comunicare in modo più efficace, per identificarsi con un particolare gruppo, per chiudere lo status gap, per esprimere la buona volontà e il sostegno (J.J. Gumperz, 1982: 144).

Un'osservazione pertinente è che anche Gumperz ha osservato che la commutazione viene provocata delle emozioni umane.

Occorre menzionare le analisi di Malik (1994), il quale distingue dieci funzioni del code-switching. Quindi, la commutazione di codice appare quando i locutori: non trovano la parola adeguata nella seconda lingua, non hanno la competenza 
comunicativa in ambedue le lingue, sono stanchi o arrabbiati, tentano di sottolineare qualcosa in modo più persuasivo, esprimono inchieste, inviti, gratitudini o semplicemente per abitudine, parlano la lingua comune ai gruppi sociali a causa dell'identificazione con essi, si rivolgono a un vasto pubblico per parlare la lingua da loro conosciuta, a causa del contesto conversazionale, quando la scelta del codice influisce sul significato e infine per attirare l'attenzione dei loro interlocutori. Va tenuto presente che anche Malik sottolineava l'importanza delle emozioni durante il code - switching.

Avendo ormai messo in luce le caratteristiche salienti dell'uso della commutazione dipendente dalle emozioni che confermano gli studi dei soprannominati studiosi, è opportuno citare Cerruti e Regis, i quali scrivevano che

l'esame della vasta gamma di funzioni del CS italiano-dialetto mostra dunque come l'uso alternato dei due codici sia una pratica ben diffusa, largamente accettata e anche automatizzata nella conversazione (M. Cerruti, R. Regis, 2005: 186).

Volendo succintamente riepilogare l'essenziale di quanto esposto fin qui, si può osservare che non esistono delle regole, quando viene usata la commutazione, anzi, essa dipende da vari fattori, tra i quali il cambio d'argomento, d'interlocutore, di intenzioni e, infine, l'aspetto emotivo. Le suddette constatazioni saranno il punto di riferimento per l'analisi del corpus linguistico.

\section{Dialetto talamonese}

Il dialetto talamonese è una variante locale del dialetto valtellinese che appartiene ai dialetti lombardi. Valsecchi Pontiggia (1990: 9) scriveva che "racchiude in sé le caratteristiche del gruppo occidentale (milanese e limitrofi) e del gruppo orientale (bergamasco, bresciano e limitrofi) con infiltrazioni ladine, specialmente nell'alta valle, e germaniche". Il dialetto talamonese è stato analizzato da padre Abramo Bulanti, il quale da molti anni raccoglie le parole dialettali e infine nel 1990 ha scritto la prima edizione del dizionario talamonese: U1 talamùn, Vocabolario Talamonese, riedita nel 2014, arricchita di 300 parole, disponibile anche online.

Le parole nel dialetto talamonese sono di provenienza latina a causa dell'insediamento romano, ma si nota anche l'influenza dei popoli che ci hanno vissuto, come Etruschi, Reti, Orobi, Longobardi, Francesi, Grigioni (Galanga, 1992; Turazza, 1920; Bulanti, 1990). Tante persone sono emigrate in Svizzera, in Francia, negli Stati Uniti, in America Latina, in Australia, ma anche nelle città italiane più 
importanti, ossia Roma, Venezia, Livorno e poi sono ritornate, portando delle parole da diverse lingue (D. Larraburu, 2008). L'italianizzazione arrivò a Talamona più tardi che nel resto dell'Italia, aiutando il dialetto a conservare la sua forma più antica.

Talamona è un paese abbastanza piccolo per il fatto che occupa soltanto una superficie di 21,24 kmq. Vicino si trovano altri paesi piccoli, i quali, non essendo dominanti, non influenzano il dialetto talamonese. Le grandi città sono distanti e inoltre, Talamona a nord confina con il fiume Adda e a sud con le Alpi Orobie. Bulanti afferma che "era una comunità contadina chiusa e lontana dalle vie di comunicazione, così ha potuto conservare a lungo la tipicità del suo dialetto" (A.M. Bulanti, 1994: 4). Occorre mettere in evidenza anche che Talamona si trova in alto sull'apice del conoide di deiezione dei due torrenti la Roncaiola e il Tartano. Allora, Talamona sempre fu poco abitata, secondo i dati del 2014 ci abitavano 4722 persone. Grazie agli studi di Larraburu (2008), Turazza (1920), Riva (2000) si può affermare che il paese è da sempre poco abitato. Più il paese è piccolo, meno gente ci abita, più conservato è il dialetto. Se sommiamo le annotazioni qui esposte, possiamo arrivare alle seguenti conclusioni: Talamona è situata in un posto difficile da raggiungere in passato per vivere e per questo motivo, il talamonese fu meno influenzato dall'italianizzazione. In più, è un idioma che ha avuto delle condizioni favorevoli in passato per mantenersi più a lungo la cosiddetta parlata locale.

\section{Emozioni che provocano l'uso del dialetto}

Avendo finora spiegato il fenomeno del cambio di codice, il modo in cui si comprendono le emozioni e le basi del dialetto talamonese, si passa alle situazioni in cui i talamonesi hanno elencato i cambi di codice in dialetto dovuti alle emozioni.

Il corpus linguistico proviene dalla ricerca svolta tramite i colloqui semidirettivi e questionari sociolinguistici con gli abitanti di Talamona. L'analisi è stata svolta nel settembre 2014, marzo 2015, settembre 2016. Per le analisi sono state scelte le persone di diversa età, sesso, istruzione, abitanti di Talamona da molti anni che svolgevano diversi lavori e arrivavano dai diversi gruppi sociali. La ricerca comprendeva 12 colloqui semidirettivi e 22 questionari sociolinguistici. Ai colloqui hanno partecipato 3 fonti giovani di età meno di 35 anni, 5 fonti adulte di età tra i 35 e 60 anni, e 4 fonti anziane oltre 60 anni. Nei questionari hanno partecipato 5 fonti giovani, 9 fonti adulte e 8 anziane. I risultati dell'analisi hanno dimostrato che i talamonesi compiono un'alternanza di codice per la specifica funzione dell'idioma, per l'abitudine linguistica o per la competenza comunicati- 
va maggiore in una delle due varietà, e il cambio di codice per la funzione, per l'abitudine, per la competenza, e per esprimere le emozioni. Adesso si tenta di ampliare la ricerca, ponendo enfasi non sull'analisi cognitiva svoltasi tramite il profilare delle condizioni di felicità degli atti linguistici, ma sull'aspetto emotivo del cambio di codice.

\section{Emozioni negative}

Di seguito si tratterà degli esempi della commutazione di codice che sono provocati dalle emozioni negative, come la rabbia, l'impazienza e l'irritazione. $\mathrm{Si}$ inizia con i volgarismi, le parolacce che spesso vengono espresse in dialetto per rabbia o irritazione, allora sono pronunciate spontaneamente.

(1) Quando la gente si arrabbia, all'improvviso tira fuori un volgarismo in dialetto "vada via al cuul!".

L'esclamazione è utilizzata dal locutore arrabbiato tramite il cambio di codice dall'italiano al dialetto. L'esempio è una commutazione di codice, perché il locutore spiega che si tratta di tirare fuori una frase in dialetto dalle frasi italiane. Vada via al cuul! significa vattene da quel posto! nel linguaggio colloquiale vaffanculo! Il dialetto, quindi, appare come una varietà nella quale il locutore parla, quando si sente arrabbiato. I volgarismi in dialetto rafforzano il comunicato, sottolineando l'emozione negativa di rabbia, d'irritazione o d'impazienza. In questo caso non si può specificare quale situazione ha preceduto tale emozione, ma sicuramente era una situazione negativa.

(2) Quando si è nervosi in dialetto si dicono le parolacce, come "vada via cuul!", o vai a quel paiis!" anche se prima si parlava in italiano.

In questo esempio, il locutore spiega che in una situazione comunicativa difficile, quando ci si sente nervosi e si vuole dire le parolacce, le si dice proprio in dialetto. Vada via cuul! ossia vaffanculo! è un'esclamazione offensiva. Vai a quel paiis! significa letteralmente vai a quel paese!, vattene!, il che di solito è provocato dal comportamento dispiacevole di un'altra persona. Questi sono gli esempi di commutazione di codice, poiché il locutore, prima e dopo, parla in italiano. Quando egli si arrabbia fortemente, sceglie all'improvviso il dialetto per esprimere le parolacce. 
(3) A volte metto una frase in dialetto. Alcune espressioni sembrano volgari, adatte per usare verso la mucca non all'uomo. Vado in un gruppo di volontariato, dove c'è un ragazzo disabile che non parla, ma scrive e scrive in dialetto quando vuole dire $i$ volgarismi.

Nel seguente esempio, il locutore è disabile, precisando, muto, e comunica con gli altri scrivendo le frasi su un foglio. Quando egli diventa arrabbiato, scrive in dialetto le parolacce su un foglio. Nella mente del locutore, le parolacce sono legate all'idioma dialettale. Pure in questo caso, si osserva una commutazione di codice, perché prima o dopo il ragazzo usa l'italiano per iscritto.

(4) A volte succede che la mia amica che parla quasi soltanto in italiano quando si arrabbia, grida in dialetto le parolacce.

In questo esempio, invece, si vede che quando la locutrice si arrabbia, anche se di solito non usa il dialetto, lo sceglie per le parolacce e ciò è causato proprio dall'emozione di rabbia. Bisogna tener presente che si nota la commutazione di codice.

(5) Non parlo più con Mario. Su propri gneco! Mi sono incavolata parecchio! Su propri gneco! (femminile), su propri gnech! (maschile) — si dice proprio in dialetto per indicare in fatto di essere molto arrabbiati.

In questo esempio, una donna ha litigato con un uomo, chiamato Mario. All'inizio, la donna si esprime in italiano, poi in dialetto spiega che si è offesa; quindi, si osserva una commutazione di codice. Quando la locutrice sente la rabbia, cambia il codice al dialetto. A questo proposito occorre rimarcare il fatto che i ricordi di aver litigato con qualcuno, allora le esperienze fortemente emotive, provocano l'emozione di risentimento e in seguito, l'uso del dialetto.

(6) "Tas giù!" - la mamma grida al figlio in dialetto, mentre con la sua amica parla in italiano.

Nell'esempio seguente si vede una situazione comunicativa nella quale la madre sta parlando con una sua conoscente e suo figlio la infastidisce, poiché le interrompe così la conversazione. La donna allora grida tas giù!, che vuol dire stai zitto!, ed esegue la commutazione. Bisogna sottolineare che la donna diventa impaziente e arrabbiata per l'atteggiamento del proprio figlio e gli ordina di stare in silenzio, dicendolo in dialetto.

(7) Guarda che casino! Rema scia qui rop! Se non pulisci la camera, non esci stasera! "Rema scia qui rop" — la madre nervosa che dice alla figlia di mettere in ordine. 
Nell'esempio riportato sopra si vede che la madre chiede in dialetto alla figlia in dialetto di fare ordine in camera. Vale la pena prestare attenzione al fatto che la donna, a causa delle emozioni, allora per la rabbia e l'impazienza, passa al dialetto. Di nuovo, il comportamento di un'altra persona provoca un'emozione negativa.

(8) Cosa? Mangi già la terza brioche? Finis da maià tu podet ues scià pie! "Finis da maià tu podet ues scià pie" — la mamma dice al figlio di smettere di mangiare perché può essere già pieno.

La madre consiglia al figlio di finire di mangiare e glielo dice in dialetto tramite la commutazione: Smettila, di mangiare perché sei pieno. È un rimprovero per il comportamento del figlio. Particolare attenzione si dovrà rivolgere al fatto che l'emozione di impazienza e la preoccupazione provoca l'uso del dialetto.

(9) Non ti compro quel gelato. Piantelo da fa i nuc! O Dio, come sei pesante oggi. „Piantelo da fa i nuc!" - smettila di fare i capricci. Si dice al bambino che piange perché non ottiene quello che vuole.

Il cambio di codice avviene dall'italiano al dialetto. La locutrice è una donna che rimprovera il figlio. Dice in dialetto: piantelo da fa $i$ nuc, che significa non fare $i$ capricci. Di centrale importanza è che le emozioni negative di impazienza e di rabbia causate dal comportamento del bambino provocano il cambio di codice al dialetto.

(10) Calmati immediatamente! Se tu la smetet minga da fa ul bambu te du un crapadum! Non sto mica scherzando! "Se tu la smetet minga da fa ul bambu te du un crapadum" - la mamma si rivolge al figlio se non la smette da fare lo scemo, gli dà una sberla.

La madre sgrida il figlio per il suo comportamento e dice in dialetto: se non smetti di fare lo stupido, ti do una sberla. È così nervosa che perfino minaccia di picchiarlo. Prima ella parla in italiano e poi in dialetto, per poi continuare in italiano: si ha quindi a che fare con una commutazione di codice.

(11) Il dialetto si fa capire al volo, certe cose hanno più effetto, ad esempio quando qualcuno ci innervosisce dice in dialetto "Noiem ciu!". Si parla in modo più forte che in italiano.

Noiem ciu vuol dire non annoiarmi. Il locutore lo dice, quando sente noia, delusione, fastidio, impazienza e vuole liberarsi dall'irritante presenza di qualcuno. La fonte spiega che l'uso del dialetto enfatizza il significato che sembra più persuasivo al destinatario. 
(12) Lasciami stare! Destot! Non voglio uscire con te! Non mi va! "Destot!" Togliti! Detto quando qualcuno è d'impiccio.

Destot in italiano si può spiegare come lasciami stare, non annoiarmi, vattene. Lo si può usare, quando qualcuno ci dà fastidio, è insopportabile. Si osserva nell'esempio che la frase in dialetto è tra le fasi italiane; quindi, si ha a che fare con la commutazione.

(13) Ma come sei pesante! Rump minga! Non ho voglia di mangiare la pizza stasera e basta. "Rump minga!" - non rompere! non stressare! Quando una persona ci dà fastidio.

Rump minga allora non stressarmi, non annoiarmi si rivolge alla persona stancante, pesante, che ci stressa, ci infastidisce e risulta che di nuovo il comportamento altrui provoca emozioni negative e in seguito, l'uso del dialetto.

(14) Dai, Carlo, perché mi chiedi il prezzo della borsa? Laghetul giuà! L'ho presa con i miei soldi e poi adesso ci sono pure i saldi! "Laghetul giuà" — la moglie arrabbiata lo dice al marito di non interessarsi o lasciare perdere per quello che sta facendo o dicendo.

Laghetul giuà, che significa lascia perdere, costituisce un altro esempio del cambio di codice, questa volta, detto dalla moglie verso il marito. Si presuppone che il marito abbia rimproverato la moglie di spendere troppo, e lei gli ha risposto, irritata, cambiando la lingua in dialetto. Ne consegue che il comportamento del marito ha provocato emozioni negative nella donna e in seguito, l'uso del dialetto.

Negli esempi seguenti, il locutore sente ansia, la quale causa l'emozione di spavento, il che influisce sull'uso del dialetto.

(15) La madre sta facendo la spesa al mercato quando suo figlio corre sulla strada: Fabio! Dove sei? Che stremizzi! Vieni qua! "Che stremizzi!" - che spavento! Quando senti un rumore improvviso o vedi qualcosa che ti spaventa.

Quando uno si spaventa, è difficile controllare la lingua. Il locutore compie il cambio di codice in modo inconscio. In questo esempio, una donna fa la spesa al mercato e all'improvviso vede che il suo bambino scappa e corre verso la strada. Si deve al riguardo constatare che l'atteggiamento del bambino suscita un'emozione di grande spavento nella donna e provoca il cambio di codice in dialetto.

(16) È caldo questo ferro da stiro! Tuca minga! Ti farà male! „Tuca minga!” La mamma preoccupata rivolta al figlio ,non toccare!” 
In questo esempio si vede una madre molto spaventata e preoccupata per la salute del proprio figlio. Lo avverte, gridando in dialetto di non toccare il ferro da stiro. Prima gli informa in italiano che il ferro da stiro è caldo e poi grida tuca minga!, che significa non toccare! Le madri di solito si sforzano di parlare l'italiano con i propri figli, perché è la lingua standard usata a scuola. Da ciò risulta chiaro che la donna sente lo spavento per il comportamento del proprio figlio e perciò usa il dialetto in modo inconscio.

(17) Dammi la mano che c'è la strada! Sta fermu! O no! „Sta fermu!” - la mamma preoccupata grida di stare fermo al figlio che sta correndo sulla strada.

Nell'esempio seguente, la madre si rivolge in dialetto al proprio figlio che con il suo atteggiamento suscita ansia nella madre. Il bambino corre sulla strada, il che è molto pericoloso. La donna grida: Sta fermu!, che significa stai fermo! ed è l'esempio della commutazione di codice. Da ciò che è stato precedentemente detto si evince che la forte ansia e lo spavento provocano l'uso del dialetto.

(18) Che ragno gigante! Iösus Maria! Buttalo fuori! "Iösus Maria!" - diciamo quando ci spaventiamo o stupiamo ad esempio per la grandezza del ragno.

In tante lingue c'è l'abitudine di gridare chiamando nomi dei santi, quando si è nervosi, scioccati, spaventati. Il locutore vede un ragno e si spaventa; spaventato fa una commutazione in dialetto.

Adesso vengono presentati degli esempi nei quali il locutore sente le emozioni negative come il dispiacere, la tristezza.

(19) Portami l'acqua naturale, cara. Su sträca. Quando si è anziani, si è anche stanchi. "Su sträca" - la nonna si lamenta di essere stanca in dialetto.

La donna anziana dice su sträca, che significa in dialetto: sono stanca. Il cambio dalla lingua italiana al dialetto e poi il ritorno all'italiano è l'esempio del cambio di codice. La condizione fisica della donna provoca il passaggio al dialetto.

(20) Non posso venire da voi. El mè fa mäl ul scagnel. Devo riposare. "El mè fa mäl ul scagnel" - il nonno si lamenta dei dolori all'anca.

In questo esempio un anziano afferma che sente il dolore e lo dice in dialetto. Lo stato fisico del corpo umano influisce sulle emozioni umane come dispiacere, tristezza e il comunicato viene detto in dialetto tramite la commutazione. 
(21) Non mi aspettare. El mè mänca ul fiaa. Arrivo piano da solo. "El mè mänca ul fiaa" - il nonno fa fatica a respirare.

El mè mänca ul fiaa significa mi manca il fiato ed è un'espressione detta da una persona anziana. L'atto di lamentare per il proprio stato fisico suscita emozioni negative e conduce all'uso del dialetto da parte della persona anziana.

(22) Non ci vado con te al parco giochi. Su stufa marscia. Magari lo zio viene. "Su stufa marscia”. La zia lo dice alla nipote che è molto stanca.

La donna dice su stufa marscia, che vuol dire sono molto stanca, e in quel modo spiega alla nipote che non può dedicarle il suo tempo per giocare insieme. Un'altra volta lo stato fisico ha provocato la stanchezza e di conseguenza, l'uso della varietà dialettale.

(23) Non volevo farti male. El me suris. Dai, facciamo pace. "El me suris" - mi dispiace. Si esprime il proprio dispiacere per una particolare situazione.

Il locutore chiede scusa a qualcuno. Con l'atto di scusare el me suris, che significa mi dispiace, dimostra che sente i rimorsi e la tristezza; ciò ha provocato l'uso del dialetto.

\section{Emozioni positive}

Ora si passa alle emozioni positive, iniziando con l'empatia.

(24) Gli è morta un mese fa la moglie. Ha perso anche il lavoro. U perdu tut. Non avevano neanche dei figli. "U perdu tut" — lui ha perso tutto.

Il locutore presenta la situazione di un amico che nello stesso tempo ha perso la moglie e il lavoro, quindi ha avuto molte disgrazie. Quando parla della situazione dell'amico, passa al dialetto, eseguendo la commutazione. Il locutore sente l'empatia verso il locutore e in seguito passa al dialetto.

(25) Quando sono tornata per comprare quel vestito, già non c'era più. Paziensa. Compro poi un altro, più bello. "Paziensa" - Pace. Pazienza. Esprime rassegnazione o sollievo. 
La donna afferma che voleva comprare un vestito, ma quando è arrivata al negozio per comprarlo, esso non c'era più, e poi come se volesse consolarsi dice in dialetto paziensa, che significa pazienza in italiano, facendo la commutazione. Sembra che la donna si stia consolando in questa situazione che le è capitata.

(26) Dai, è vero che Giovanni si è comportato male con te. Laghimula boi. Trovi poi un altro. "Laghimula boi" - due persone stanno discutendo e una consiglia all'altra di lasciar perdere, perché la vuole calmare.

Nell'esempio che segue il locutore sta consolando un'altra persona. Laghimula boi, dunque lascia perdere, è un consiglio di smettere di preoccuparsi. Questa espressione viene detta per tranquillizzare qualcuno, a causa della empatia.

(27) Lo so che sei stressata che non riesci a rimanere incinta. Laghetul giua. Abbia fiducia in Dio. "Laghetul giua" - due donne discutono e una dice all'altra "non preoccuparti".

Una donna consola un'altra che non può avere bambini. Ella le dice: laghetul giua, che vuol dire non preoccuparti. Quando si parla delle questioni intime, sentendo la compassione, l'empatia e la consolazione, si usa il dialetto.

(28) Anche se Maria non è più tua amica, ci sono ancora io. Laghetul giuà. "Laghetul giuà" - espressione tipicamente talamonese che invita a non preoccuparsi più di tanto.

Anche in questo esempio la locutrice consola una donna, la quale ha perso una sua amica. Si notano l'empatia, la volontà di consolare un'altra persona, le emozioni che in seguito provocano l'uso del dialetto.

(29) Lo so che sei molto triste, Maria. Ma ricordati ... Mei insci ca pesc. "Mei insci ca pesc" - meglio così che peggio. Si dice a una persona che va vissuto una brutta esperienza ricordandola perché poteva andare peggio.

Il locutore vuole consolare qualcuno e ciò provoca l'uso dialettale. Bisogna mettere in risalto il fatto che la consolazione, l'empatia e la compassione vengono espresse tramite una commutazione di codice dall'italiano al dialetto.

(30) Baby-sitter calma il bambino: O mamma mia ti sei rotto il ginocchio? Ricordati un vecchio detto talamonese: Per mingo sentì dulur strensc i denc' è serà i occ'. Vado a chiamare tua madre. "Per mingo sentì dulur strensc $i$ denc' è serà i occ"' - detto talamonese: per non sentire dolore stringi $i$ denti e chiudi gli occhi. 
La donna, per consolare il bambino, dice un detto di origine talamonese. Stringere i denti e chiudere gli occhi, quando ci si sente male, sono i modi che conducono a calmare e consolare il bambino. La donna condivide lo stato emotivo del bambino.

(31) Non è successo niente. Lamentes mingo... Dai ... è solo un piccolo graffio. "Lamentes mingo" - il bambino si è fatto male, piange, e la mamma lo vuole consolare e gli dice di non lamentarsi.

La madre tenta di consolare il figlio, il quale si è fatto male e perciò è triste. La madre gli dice di smettere di piangere in dialetto: lamentes mingo significa infatti non lamentarti. La madre cerca di curare il figlio, di consolarlo e proprio questa sua attitudine provoca l'uso del dialetto.

Si procede con esempi del cambio di codice provocato da altre emozioni positive, come ad esempio simpatia, meraviglia.

(32) Ma cos'hai combinato, eh? Asnun! Vieni qua! Asnun! - dopo che il ragazzo ha fatto una cavolata, il papà gli dà dell'asinone, cioè gli dice grande asino, testardo.

Il padre chiama suo figlio asnun, che vuol dire testardo, monello, mentre si divertono insieme. È un rimprovero positivo usato nell'ambito famigliare: il padre scherza con il figlio in dialetto. Va tenuto presente che il padre sente simpatia e per questo scherza con il figlio in dialetto.

(33) Ma hai mangiato tutte le caramelle! Bruttu mustru! Golosone! Non hai lasciato niente per la nonna! Bruttu mustru! - la nonna rimprovera il nipotino dandoli del mostriciattolo in modo benevolo.

In questo esempio, invece, la nonna rimprovera il nipote con simpatia, con amore, nel senso positivo, benevolo, dicendo in modo spiritoso bruttu mustru, che vuol dire monello. Le emozioni positive che prova la nonna provocano il cambio di codice in dialetto. Nel talamonese, la parola mustru assume anche gli altri significati, il che mostra un altro esempio.

(34) Sei riuscito a vincere quel concorso? Che mùstru! Complimenti! "Che mùstru!" - Che furbone nel senso che bravo!

L'esclamazione che mùstru!, cioè che bravo! che furbo!, viene usata in questo esempio di commutazione di codice perché il locutore ammira il talento del vincitore di un concorso e gli sta facendo i complimenti. Le emozioni di stupore 
e di meraviglia causate dal comportamento altrui provocano l'uso spontaneo del dialetto.

(35) Ma hai sentito che lui ha visto questa gara? L'è propri un mùstru! "L'è propri un mùstru!" - lui è un vero fenomeno!

Rimanendo ancora con l'espressione dialettale mustru, si cita l'espressione l'è propri un mùstru!, la quale significa lui è un vero fenomeno! Il locutore si meraviglia, il che provoca l'uso del dialetto.

(36) Che bella questa ma cchina! Iööö! Non ci credo che sia di Paolo! "Iööö”espressione di meraviglia.

Iöö, che vuol dire o mamma mia, è l'ultima espressione che si vuole presentare. È un esempio della commutazione di codice perché avviene tra le frasi italiane. Proprio le forti emozioni positive come la meraviglia provocano l'uso del dialetto.

\section{Conclusioni}

Volendo succintamente riepilogare l'essenziale di quanto esposto fin qui, si può osservare che quando un italiano che conosce oltre all'italiano anche il dialetto, sente le forti emozioni positive o negative, spesso sceglie il dialetto. Da quel che si è visto, un italiano arrabbiato dice spontaneamente le parolacce in dialetto. Inoltre, di solito si pensa che gli italiani, i quali non usano il dialetto ogni giorno, non iniziano spontaneamente a dire le parolacce in dialetto. L'esempio (4) ci ha dimostrato invece che le parolacce in dialetto le pronunciano anche le persone, le quali nelle situazioni quotidiane non usano spesso il dialetto. Ciò succede perché la varietà dialettale serve a esprimere emozioni in modo più forte, più efficace. Comunemente si crede che le parolacce possano essere dette in dialetto non nel modo cosciente, intenzionale, e nella maggior parte dei casi lo è, ma nell'esempio (3), la persona disabile comunica scrivendo, quindi ha più tempo per riflettere, quando sente la rabbia, sceglie pure di scrivere in dialetto, allora apposta sceglie il dialetto per rafforzare la sua espressione.

È importante enfatizzare anche che il cambio di codice dall'italiano al dialetto (ciò riguarda anche le altre nazioni che hanno nel loro paese la lingua ufficiale e i dialetti) per il motivo delle emozioni negative, come la rabbia, il risentimento, l'impazienza e il fastidio, non si verifica soltanto tramite i volgarismi. Queste emozioni evocano diverse commutazioni di codice al dialetto e nella maggior parte, le emozioni sono provocate dal comportamento dei bambini $(6,7,8,9,10)$, 
dal comportamento altrui che ci infastidisce $(11,12,13,14)$ o da ricordi emotivi negativi (5).

Si può anche affermare riassuntivamente che i locutori talamonesi, quando sentono ansia, usano all'improvviso la varietà dialettale. Di solito, quando si è scioccati o fortemente spaventati, non si controlla la propria lingua e perciò questo cambio dovuto all'ansia non sia intenzionale, pianificato, ma inconscio. Vengono notati degli esempi, soprattutto quelli in cui i comportamenti dei figli hanno spaventato le loro madri $(15,16,17)$ e quando un animale ha spaventato un uomo (18).

Da ciò che è stato precedentemente detto si evince anche che gli italiani eseguono il cambio di codice per il fatto di sentire tristezza, dispiacere, stanchezza. Il motivo di sentire queste emozioni è il debole stato fisico del corpo umano (19, 20, 21, 22) o la volontà di scusare qualcuno (23). Avendo dei problemi corporei, i locutori si sono sentiti anche male dal punto di vista emotivo.

È da segnalare inoltre che il dialetto viene usato, quando il locutore pratica l'empatia per le disgrazie altrui (24), per consolare se stesso (25) o qualcuno (26, $27,28,29)$. Vale la pena aggiungere che negli esempi analizzati le donne volevano consolare in dialetto i loro bambini che si sono fatti male $(30,31)$, anche se di solito, le donne tentano di usare solo la lingua italiana con i figli per evitare problemi scolastici.

Per di più, anche le emozioni positive di simpatia hanno provocato l'uso del dialetto, ad esempio, mentre gli adulti scherzavano con i loro nipoti $(32,33)$ e quando si esprimeva meraviglia, ammirazione per i risultati ottenuti da qualcun altro $(34,35,36)$. Inoltre, si può osservare che esprimere in dialetto le emozioni è un atto spontaneo, non pianificato e non intenzionale. Pronunciare alcune espressioni in dialetto, le rende più forti, il loro significato è più forte ed espressivo secondo i locutori.

Dall'analisi risulta che di solito, con il cambio di codice i talamonesi esprimono più spesso le emozioni negative che positive. A volte le famiglie italiane non usano mai il dialetto e non lo tramandano ai figli. A volte ci sono famiglie nelle quali ci sono più dialetti e per non mischiare i dialetti non li usano, ma cercano di usare una lingua comune, ossia l'italiano, soprattutto per insegnare ai bambini la lingua standard. L'uso del dialetto varia nel Nord e nel Sud del paese. Però, usare la varietà dialettale per esprimere le emozioni positive e negative è un fenomeno comune e molto interessante che si cercava di dimostrare dagli esempi di cambio di codice tra l'italiano e il dialetto talamonese. 


\section{Riferimenti citati}

Alfonzetti, G. (1992). Il discorso bilingue. Italiano e dialetto a Catania. Milano, Franco Angeli.

Berruto, G. (1985). 1 pulman 1-è nen ch-a cammina tanto forte. Su commutazione di codice e mescolanza dialetto - italiano. Vox Romanica, 44, 59-76.

Berruto, G. (1989). On the Tipology of linguistic Repertories. In U. Ammon (Ed.), Status and Function of Languages and Language Varietes (pp. 552-569). Berlin - New York, De Gruyter.

Berruto, G. (1990). Italiano regionale, commutazione di codice e enunciati mistilingui. In M. A. Cortelazzo \& A. M. Mioni (a cura di), L'italiano regionale (pp. 105-130). Roma, Bulzoni.

Bettoni, C., \& Rubino, A. (1996). Emigrazione e comportamento linguistico. Un'indagine sul trilinguismo dei siciliani e dei veneti in Australia. Galatina, Congedo.

Bulanti, A. M. (1990). Ul talamùn. Vocabolario Talamonese. Talamona, I Soci dè la Crüsco dè Talamuno.

Bulanti, A. M. (2014) Ul talamùn. Vocabolario Talamonese (2a ed.). Talamona, I Soci dè la Crüsco dè Talamuno.

Cerruti, M., \& Regis, R. (2005). “Code-Switching” e la teoria linguistica: la situazione italo-Romanza. Rivista di Linguistica, 17(1), 179—208.

Dąbrowski, A. (2014). Czym są emocje? Prezentacja wieloskładnikowej teorii emocji. Analiza i Egzystencja, 27, 123-146.

Dykta, D. (2021). Ciao! Me uala? Perché i talamonesi cambiano il codice. Analisi cognitiva dell'alternanza e del cambio di codice tra l'italiano e il dialetto talamonese. Katowice, Wydawnictwo Uniwersytetu Śląskiego.

Ekman, P. (2012). Emocje ujawnione. Odkryj, co ludzie chca przed toba zataić, i dowiedz się czegoś więcej o sobie (W. Białas, Przeł.). Gliwice, Helion.

Galanga, E. E. (1992). Sintesi di storia della Valtellina medio-alta. Sondrio, Museo Etnografico Tiranese.

Gobber, G., \& Morani, M. (2014). Norma, lingua e uso della lingua. In G. Gobber \& M. Morani (a cura di), Linguistica generale. Milano, Mc Graw. https://studylibit. com/doc/6434775/norma--linguaeuso-della-lingua (accesso: 26.04.2021).

Grassi, C., Sobrero, A. A., \& Telmon, T. (1997, 2001, 2012). Fondamenti di dialettologia italiana. Roma - Bari, Editori Laterza.

Grassi, C., Sobrero, A. A., \& Telmon, T. (2010). Introduzione alla dialettologia italiana. Roma - Bari, Editori Laterza.

Gumperz, J. J. (1982). Conversational Code-Switching. In J. J. Gumperz (Ed.), Discourse Strategies (pp. 59-99). Cambridge (England), Cambridge University Press.

ISTAT, (2017). L'uso della lingua italiana, dei dialetti e di altre lingue in Italia. https:// www.istat.it/it/archivio/207961 (accesso: 28.04.2021).

Larraburu, D. (2008). Talamonesi nel Mondo. Trenta giorni di nave a vapore. Sondrio, Polaris.

Malik, L. (1994). Sociolinguistics: A Study of Code-Switching. New Delhi, Anmol. 
Riva, I. (2000). Talamona "la chiesa del paese” Parocchia Natività di Maria Vergine Talamona. Sondrio, Bettini.

Sobrero, A. A., \& Miglietta, A. (2009). Introduzione alla linguistica italiana. Roma Bari, Laterza.

Turazza, G. (1920). Talamona nella Valtellina. Notizie documentate politico-religiose. Sondrio, Società Arti Grafiche Valtellinesi.

Valsecchi Pontiggia, L. (1990). Saggio di Vocabolario Valtellinese. Sondrio, Bissoni Editore. 